Provided by the author(s) and NUI Galway in accordance with publisher policies. Please cite the published version when available.

\begin{tabular}{|c|c|}
\hline Title & $\begin{array}{l}\mathrm{A} \mathrm{Cu}(\mathrm{II}) \text { complex targeting the translocator protein: in vitro } \\
\text { and in vivo antitumor potential and mechanistic insights }\end{array}$ \\
\hline Author(s) & $\begin{array}{l}\text { Montagner, D.; Fresch, B.; Browne, K.; Gandin, V.; Erxleben, } \\
\text { Andrea }\end{array}$ \\
\hline $\begin{array}{l}\text { Publication } \\
\text { Date }\end{array}$ & 2016-12-01 \\
\hline $\begin{array}{l}\text { Publication } \\
\text { Information }\end{array}$ & $\begin{array}{l}\text { Montagner, D., Fresch, B., Browne, K., Gandin, V., \& } \\
\text { Erxleben, A. (2017). A Cu(ii) complex targeting the } \\
\text { translocator protein: in vitro and in vivo antitumor potential } \\
\text { and mechanistic insights. Chemical Communications, 53(1), } \\
\text { 134-137. doi: } 10.1039 / \text { C6CC08100B }\end{array}$ \\
\hline Publisher & Royal Society of Chemistry \\
\hline $\begin{array}{l}\text { Link to } \\
\text { publisher's } \\
\text { version }\end{array}$ & http://dx.doi.org/10.1039/c6cc08100b \\
\hline Item record & http://hdl.handle.net/10379/7199 \\
\hline DOI & http://dx.doi.org/10.1039/c6cc08100b \\
\hline
\end{tabular}

Some rights reserved. For more information, please see the item record link above. 


\title{
A Cu(II) Complex Targeting the Translocator Protein: In Vitro and In Vivo Antitumor Potential and Mechanistic Insights ${ }^{\dagger}$
}

\author{
D. Montagner, ${ }^{a, b}$ B. Fresch, ${ }^{c, d}$ K. Browne, ${ }^{a}$ V. Gandin*e and A. Erxleben*a \\ ${ }^{a}$ School of Chemistry, National University of Ireland, Galway, Ireland \\ ${ }^{b}$ Department of Chemistry, National University of Ireland, Maynooth, Ireland \\ ${ }^{c}$ Department of Chemistry, University of Liège, Liège, Belgium \\ 'Dipartimento di Scienze del Farmaco, Universita' degli Studi di Padova, Padova, Italy
}

\begin{abstract}
A new Cu-based anticancer metallodrug which targets the translocator protein is reported. [CuBr2(TZ6)] elicits a remarkable in vitro cytotoxicity in sensitive and multidrug resistant cell lines and induces a $98 \%$ reduction of tumor mass in a murine tumor model. Target binding was studied by experimental and computational methods.
\end{abstract}

The translocator protein (TSPO) is a relatively small transmembrane protein (18 kDa) that is mainly located at the outer mitochondrial membrane. ${ }^{1-3}$ It is overexpressed in certain cancerous tissues in which its expression correlates with disease progression and malignant behavior..$^{4-7}$ The TSPO plays an important role in mitochondrial biochemistry, ${ }^{8,9}$ transport of heme precursors into the mitochondria, ${ }^{10}$ mitochondrial quality control and the regulation of mito-chondrial energy metabolism. ${ }^{11} \mathrm{~A}$ regulatory role for TSPO in mitochondria-mediated cell death pathways seems to arise from its complex crosstalk across multiple distinct downstream cell processes involving the mitochondria. In particular, TSPO acts as a molecular interplay linked to reactive oxygen species (ROS) signaling and its inhibition has been demonstrated to lead to cellular redox homeostasis imbalance and ultimately to cell death. The possibility to induce cancer cell death combined with the overexpression of TSPO in cancerous tissues makes TSPO a new and interesting target for metal-based chemotherapeutic agents. ${ }^{12}$ Different classes of ligands have been shown to bind to TSPO (Fig. S1, ESI). ${ }^{13-16}$ Jaremko et al. recently determined the structure of the TSPO-PK11195 adduct and the binding position of PK11195. ${ }^{17}$ Denora et al. and Trapani et al. reported detailed structure-activity relationship studies for the imidazopyridine ligand alpidem. ${ }^{18,19}$ The alpidem analogue TZ6, composed of an imidazopyridine moiety, a thiazole ring and a N,N-dialkylacetamide side chain (Fig. 1), was reported by Natile and coworkers who synthesized its Pt(II) complex. ${ }^{20,21}$ The complex had a high receptor affinity, but was poorly water soluble and less cytotoxic than cisplatin.

Complexes based on $\mathrm{Cu}$ receive significant current attention as possible anticancer drugs and numerous complexes with interesting antitumor activity have been synthesized on the assumption that endogenous metal ions are less toxic to normal cells than non-endogenous metals. ${ }^{22}$ We herein report that a highly effective and selective new anticancer agent is obtained, when the TSPO binding ligand TZ6 is combined with Cu(II) as a redox active metal center and potent ROS producer.
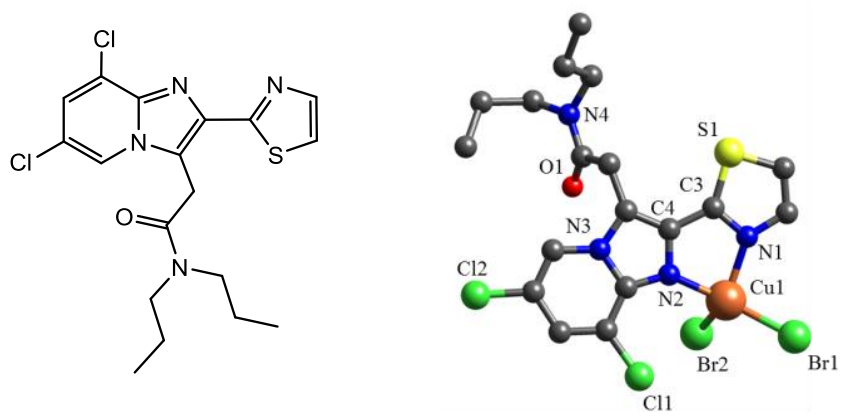

Fig. 1 Left: Chemical structure of the ligand TZ6. Right: X-ray structure of 1 . For the sake of clarity $\mathrm{H}$ atoms are omitted and only one set of the disordered isopropyl groups is shown. 
The complex $\left[\mathrm{CuBr}_{2}(\mathrm{TZ6})\right]$ (1) was obtained by reacting $\mathrm{CuBr}_{2}$ with $\mathrm{TZ6}$ at $40{ }^{\circ} \mathrm{C}(\mathrm{ESI})$. Slow evaporation of a $\mathrm{CH}_{3} \mathrm{CN}$ solution of 1 gave greenish-red, $\mathrm{X}$-ray suitable crystals. The $\mathrm{X}$-ray structure presented in Fig. 1 shows the expected bidentate nitrogen coordination of the TZ6 ligand. Two additional bromide ligands give rise to a severely distorted tetrahedral coordination sphere. Because of the small bite angle of the ligand, the N1-Cu1-N2 angle is rather acute (80.9(4) ${ }^{\circ}$ ).

Table 1 Cytotoxicity data

\begin{tabular}{|c|c|c|c|c|c|c|c|c|}
\hline & \multicolumn{8}{|c|}{$\mathrm{IC}_{50}(\mu \mathrm{M}) \pm \mathrm{SD}{ }^{a}$} \\
\hline & A549 & HCT-15 & BxPC3 & A375 & A431 & MCF-7 & A2780 & A498 \\
\hline 1 & $1.12 \pm 0.57$ & $3.16 \pm 1.24$ & $0.33 \pm 0.07$ & $2.22 \pm 0.84$ & $0.89 \pm 0.54$ & $0.21 \pm 0.01$ & $0.65 \pm 0.08$ & $1.16 \pm 0.16$ \\
\hline TZ6 & $>30$ & $>30$ & $>30$ & $>30$ & $>30$ & $>30$ & $>30$ & $>30$ \\
\hline CDDP & $10.56 \pm 1.34$ & $11.32 \pm 1.51$ & $6.17 \pm 1.37$ & $3.11 \pm 0.98$ & $1.65 \pm 0.51$ & $7.60 \pm 0.21$ & $2.23 \pm 0.39$ & $15.68 \pm 2.48$ \\
\hline OXP & $1.67 \pm 0.96$ & $1.04 \pm 0.67$ & $4.15 \pm 1.07$ & $6.30 \pm 2.01$ & $3.06 \pm 0.88$ & $3.36 \pm 1.69$ & $2.45 \pm 0.68$ & $7.91 \pm 2.42$ \\
\hline
\end{tabular}

By contrast, the Br2-Cu1-N1 (131.0(3) ${ }^{\circ}$ ) and Br1-Cu1-N2 (151.3(3) ${ }^{\circ}$ ) angles are significantly larger than the ideal $109.5^{\circ}$ angle. The TZ6 ligand is essentially planar with a dihedral angle of 5.0(15) ${ }^{\circ}$ between the thiazole ring and the imidazopyridine moiety (Table S2, ESI).

The in vitro antitumor potential of TZ6 and 1 was evaluated in cell lines representative of lung (A549), colon (HCT-15), pancreatic (BxPC3), cervical (A431), breast (MCF-7), ovarian (A2780) and kidney (A498) cancers, along with melanoma

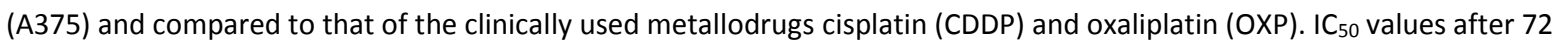
$\mathrm{h}$, calculated from dose-survival curves, are reported in Table 1. Uncoordinated TZ6 proved to be barely effective against all tested cultured human cancer cell lines. By contrast, the Cu complex showed a significant antiproliferative activity, with $\mathrm{IC}_{50}$ values in the low and sub-micromolar range. 1 was particularly effective against human breast MCF-7 cancer cells, with mean IC 50 values exceeding about 36 and 16 times those recorded with CDDP and OXP, respectively. Similarly, 1 elicited an in vitro antitumor activity that was about 19 - and 13 -fold better than that of CDDP and OXP against human pancreatic BxPC3 cells. The antiproliferative activity of 1 was also investigated in two human cell line pairs which had been selected for sensitivity/resistance to CDDP (ovarian cancer cells 2008/C13*) and OXP (colon cancer cells LoVo/LoVo-OXP) or belong to the multidrug-resistant (MDR) phenotype (colon cancer cells LoVo/LoVo MDR). The $\mathrm{IC}_{50}$ and RF values ( $R F=$ resistance factor, defined as the ratio between $I C_{50}$ of resistant cells and $I C_{50}$ of sensitive ones) are reported in Table S3. 1 exhibited a similar pattern of cytotoxicity both in platinum-sensitive and -resistant cells, and the RF values calculated for 2008/C13* and LoVo/LoVo-OXP cells were 14- to 24-fold lower than that of the reference platinum drug, thus indicating the absence of cross-resistance. Analogously, in colon cancer LoVo/LoVo MDR cells, complex 1 yielded RF values on average 30-fold lower than that obtained with doxorubicin, a drug belonging to the MDR spectrum. The cytotoxicity of 1 was also evaluated against non-tumor cells, namely human lung MRC-5 and colon CCD18-Co fibroblasts as well as human embryonic kidney HEK293 cells (Table S4). The preferential cytotoxicity of the newly synthesized Cu(II) complex towards neoplastic cells is confirmed by the selectivity index value $\left(\mathrm{SI}=\right.$ average $I \mathrm{I}_{50}$ toward normal cells divided by the average $\mathrm{IC}_{50}$ for the malignant cells). The SI of 1 is significantly higher than those calculated for CDDP and OXP (SI of 17.5, 1.8, and 12.6 for 1, CDDP and OXP, respectively).

To investigate whether TSPO expression in human cancer cells influences cell sensitivity to $\mathbf{1}$, we evaluated the expression levels of TSPO in four different human cancer cells, namely lung (A549), cell lines (Fig. 2A). A direct relationship $\left(R^{2}=0.82\right.$ ) between TSPO levels and the cytotoxic activities of 1 was found (Fig. 2B). Moreover, the affinity of 1 and uncoordinated TZ6 for the TSPO receptor was evaluated by measuring the ability of 1 and TZ6 to displace the reference compound $\left[{ }^{3} \mathrm{H}\right]-$ PK11195. The CU(II) complex showed an affinity comparable to that of free TZ6 (6.9 and 3.6 nM for 1 and free TZ6, respectively). These findings clearly confirm that TSPO plays a key role in the antiproliferative activity of 1. 


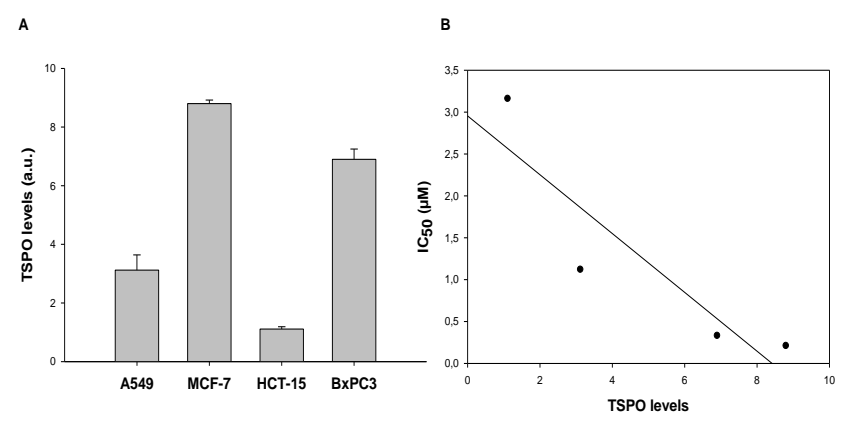

Fig. 2 A. TSPO expression in A549, MCF-7, HCT-15 and BxPC3 cells. B. Correlation between cytotoxicity and TSPO expression.

Taking into consideration previously reported experimental evidence that TSPO affects energy homeostasis and that it is implicated in the control of mitochondrial ROS production, ${ }^{11}$ we investigated the effects of 1 on the mitochondria, in particular on ROS levels, the alteration of the mitochondrial membrane potential, and the functioning of the respiratory chain. Treatment of MCF-7 cells with complex 1 induced a dose- and time-dependent increase in the cellular basal ROS production (Fig. S4A, ESI). However, the ROS production induced by 1 was lower than that provoked by antimycin A, a classical inhibitor of the mitochondrial respiratory chain at the level of complex III. An increase of cellular basal ROS production could be induced by a direct hampering effect on the respiratory chain. As depicted in Fig. S4B (ESI), 1 hampers the respiratory chain in a time- and dose-dependent manner, thus leading to a reduction of $\mathrm{O}_{2}$ consumption in treated cells. It is well known that hampering of the respiratory chain and the resulting increase of ROS production lead to the hypopolarization of the mitochondrial membrane. Consistently, a significant time and dose-dependent increase of cells with depolarized mitochondria was observed after treatment with 1 (Fig. S4C, ESI). Morphological analysis by TEM of MCF7 cells treated with the Cu complex confirmed that 1 significantly alters the mitochondria (Fig. S5, ESI).

Incubation for 12 and $24 \mathrm{~h}$ with $\mathrm{IC}_{50}$ doses of $\mathbf{1}$ induced a dramatic swelling of the mitochondria associated with decreased electron density of the inner membrane and matrix regions. The mitochondria of 1-treated MCF-7 cells showed disrupted cristae and were significantly increased in volume with respect to the mitochondria of control cells.

The increase in hydrogen peroxide production and the alteration of relevant mitochondrial pathophysiological parameters upon interaction of 1 with TSPO in human cancer cells can lead to an imbalance in the cellular redox homeostasis.

Therefore we assessed the effects induced on the sulfhydryl redox state. Fig. 3A shows the total amounts of glutathione (GSH) and oxidized glutathione (GSSG) in MCF-7 cells incubated for $48 \mathrm{~h}$ with increasing concentrations of complex 1 . While no significant changes in total cellular glutathione levels were observed, a dose-dependent increase of GSSG levels occurred. Accordingly, the Cu complex caused a dose-dependent decrease in total sulfhydryl content (Fig. 3B).
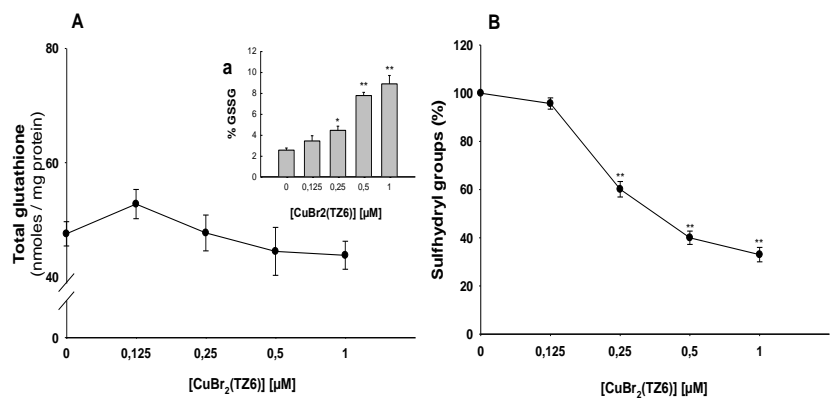

Fig. 3 Effect of 1 on the cellular redox environment. MCF-7 cells were treated for $48 \mathrm{~h}$ with increasing concentrations of 1. The amount of total glutathione (GSH + GSSG, A), oxidized glutathione (A, inset a) and sulfhydryl groups (B) were measured by means of DTNB reaction. ${ }^{*} p<0.05 ; * * p<0.01$. 
Complex 1 was evaluated for its in vivo activity in the murine LLC solid tumor. The tumor growth inhibition induced by 1 was compared to that promoted by CDDP. Seven days after tumor inoculation, tumor-bearing mice were randomized into vehicle control and treatment groups (five mice per group). Control mice received the vehicle (0.5\% DMSO (v/v) and 99.5 $\%$ of a saline solution $(\mathrm{v} / \mathrm{v})$ ), whereas treated groups received daily doses of 1 (20 and $10 \mathrm{mg} \mathrm{kg}^{-1}$ in a vehicle solution composed of $0.5 \%$ DMSO $(\mathrm{v} / \mathrm{v})$ and $99.5 \%$ of saline solution $(\mathrm{v} / \mathrm{v}))$ or cisplatin $\left(3 \mathrm{mg} \mathrm{kg}^{-1}\right.$ in saline solution). The tumor growth was estimated at day 15 and the results are reported in Table 2. As an estimation of the adverse side

effects, changes in the body weights of tumor-bearing mice were monitored every two days (Fig. S6, ESI). Noteworthy, administration of 1 induced a $c a .98 \%$ reduction of the tumor mass compared to the control group. Remarkably, the time course of body weight changes indicated that treatment with 1 did not induce significant body weight loss ( $<20 \%)$. On the contrary, the time course of body weight changes indicated that CDDP induces anorexia, with a body weight loss $>20 \%$ (Fig. S6, ESI).

Computational studies at different levels of theory (see ESI) were carried out to shed light on the molecular details of the interaction of 1 with the target TSPO protein. Fig. 4 shows the isosurfaces of constant electrostatic potential (ESP) for the TZ6 ligand and for 1.

Table 2 In vivo activity of $\mathbf{1}$ in the murine LCC solid tumor ${ }^{a}$

\begin{tabular}{lccc}
\hline Compound & $\begin{array}{c}\text { Dose } \\
\left(\mathrm{mg} \mathrm{kg}^{-1}\right)\end{array}$ & $\begin{array}{c}\text { Average tumor } \\
\text { weight (mean } \pm \\
\text { SD, } \mathbf{g})\end{array}$ & $\begin{array}{c}\text { Inhibition of } \\
\text { tumor } \\
\text { growth (\%) }\end{array}$ \\
\hline control & - & $0.457 \pm 0.08$ & - \\
$\mathbf{1}$ & 20 & $0.010 \pm 0.008^{* *}$ & 97.68 \\
$\mathbf{1}$ & 10 & $0.111 \pm 0.05^{* *}$ & 75.74 \\
CDDP & 3 & $0.038 \pm 0.03^{* *}$ & 91.75 \\
\hline
\end{tabular}

${ }^{a}$ Starting from day 7 after tumor implantation, the tested compounds were daily administered intraperitoneally. At day 15 , mice were sacrificed and tumor growth was determined as described in the ESI. ** $p<0.01$.

ESP provides information about the distribution of electron density around the nuclei of the molecule. Negative isosurfaces (red) indicate an electron-rich region. The N1-C-C-N2 site of the TZ6 ligand is characterized by a high electron density. As shown in Fig. 4, because of the presence of the bromides, a negative ESP region is also present in 1 so that the main feature of the ESP spatial distribution of TZ6 is conserved upon binding of the $\mathrm{CuBr}_{2}$ moiety. A similar spatial distribution of the molecular electrostatic potential is important in view of the binding of $\mathbf{1}$ to the TSPO target as long as the electrostatic component plays a central role in determining a favorable host-guest interaction. ${ }^{23}$ The binding of the ligands PK11195, TZ6 and of 1 to the target TSPO was first investigated by a docking procedure that samples the conformations of the ligand while keeping frozen the structure of the TSPO receptor (Rigid Docking). ${ }^{24,25}$ Subsequently, the best pose of TZ6 and three poses of 1 within the binding pocket (Fig. S8, ESI) were refined with an all-atom molecular mechanics simulation (AMBER Score) where the whole protein is flexible and the binding site can adjust its structure to the specific ligand. ${ }^{26-28}$ The values of the scoring functions are reported in Table S5 (ESI), negative values indicate favorable binding. The affinities evaluated by rigid docking of PK11195 and TZ6 for the target protein are comparable, while the interaction of 1 with the rigid binding pocket is repulsive because of the increased van der Waals radius due to the presence of the $\mathrm{CuBr}_{2}$ moiety. The AMBER Score of TZ6 is practically equivalent to the score obtained by rigid docking (Table S3, ESI). On the contrary, in the case of $\mathbf{1}$, we observe a significant rearrangement of the binding site, see Fig. 4. As a result of this induced fit process, ${ }^{26}$ the estimated binding energy of complex $1(-34.2 \mathrm{kcal} / \mathrm{mol})$ is only slightly smaller than the estimated binding energy of the free TZ6 ligand $(-51.8 \mathrm{kcal} / \mathrm{mol})$ in qualitative agreement with the relative affinity of the two molecules measured experimentally. Thus, the coplanar conformation of the imidazopyridine and thiazole rings imposed by the metal coordination does not hamper the TZ6-TSPO interaction. The orientation of $\mathbf{1}$ that results most stabilized by the structural adjustment of the binding site (structure $\mathrm{c}$ of Fig. S5, ESI) is characterized by the $\mathrm{CuBr}_{2}$ moiety pointing toward the helix containing the THR49-LEU50-ALA51 residues (TM2 following the convention of ref 17, see Fig. 4). In the best pose (Fig. 4), the TZ6 moiety of 1 (magenta) has the same orientation as the TZ6 ligand (blue) within the binding site. The channel created by the five $\alpha$-helices of the TSPO target is larger in the $\mathrm{Cu}\left(\mathrm{TZ6}_{\mathbf{B}} \mathrm{Br}_{2}\right.$-TSPO complex (light brown structure in Fig. 4) than in the TZ6-TSPO complex (light grey structure). In particular, the TM2 $\alpha$-helix of the receptor is displaced to fit the increased molecular volume of 1. In the structure of TSPO the loops between the transmembrane helices are short, with the exception of residues Gly28 to Pro45, which connect TM1 and TM2. The seven residues Glu29 to Ala35 fold into a short $\alpha$ helix that is inclined with respect to the long axis of TM1 and closes the binding pocket from the cytosolic side. The 
induced fit observed upon the binding of 1 suggests that this particular structural motif that was already found important for the binding of PK11195 17 plays indeed a key role in the modulation of the TSPO binding site.

In conclusion [ $\left.\mathrm{CuBr}_{2}(\mathrm{TZ6})\right]$ clearly targets TSPO and features significant in vitro and in vivo activity and selectivity for cancer cells over healthy cells which warrants its consideration for further development as a potential new type of anticancer metallodrug.
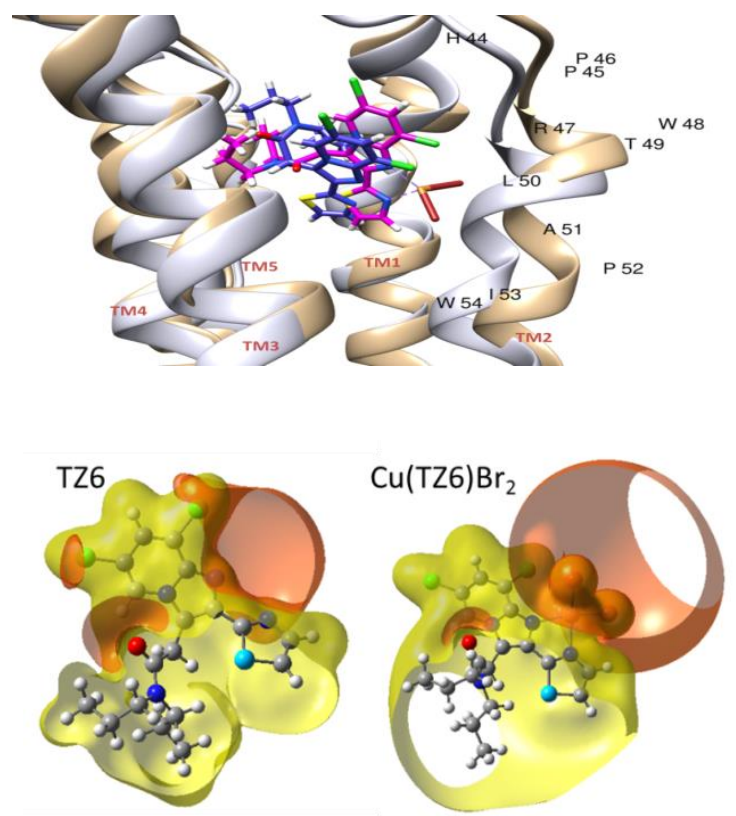

Fig. 4 Top: Isosurfaces of constant electrostatic potential (ESP). The isovalue is 0.02 a.u. Negative isosurfaces are in red and positive isosurfaces are in yellow. Bottom: Best poses obtained by the all-atom flexible docking procedure for the TZ6TSPO complex (blue ligand and light grey receptor) and for the $\mathrm{Cu}\left(\mathrm{TZ}_{\mathrm{C}}\right) \mathrm{Br}_{2}-\mathrm{TSPO}$ complex (magenta ligand and light brown receptor). The residues of the TM2 $\alpha$-helix that is displaced to bind $\mathrm{Cu}\left(\mathrm{TZ6}_{\mathrm{B}} \mathrm{Br}_{2}\right.$ are named with a one-letter code ( $\mathrm{H}$ histidine, P-proline, R-arginine, T-threonine, L-leucine, A-alanine, I-isoleucine, W-tryptophan).

\section{References}

1 C. J. D. Austin, J. Kahlert, M. Kassiou and L. M. Rendina, Int. J. Biochem. Cell Biol., 2013, 45, 1212.

2 C. Braestrup, R. Albrechtsen and R. F. Squires, Nature, 1977, 269, 702.

3 V. Papadopoulos, M. Baraldi, T. R. Guilarte, T. B. Knudsen, J.-J. Lacapère, P. Lindemann, M. D. Norenberg, D. Nutt, A. Weizman, M.-R. Zhang and M. Gavish, Trends Pharmacol. Sci., 2006, 27, 402.

4 P. Casellas, S. Galiegue and A. S. Basile, Neurochem. Int., 2002, 40, 475.

5 L. Veenman and M. Gavish, Drug Dev. Res., 2000, 50, 355.

6 K. L. Black, K. Ikezaki, E. Santori, D. P. Becker and H. V. Vinters, Cancer, 1990, 65, 93.

7 R. Nagler, O. Ben-Izhak, D. Savulescu, E. Krayzler, S. Akrish, S. Leschiner, I. Otradnov, S. Zeno, L. Veenman and M. Gavish, Biochim. Biophys. Acta, 2010, 1802, 454.

8 J. M. Bernassau, J. L. Reversat, P. Ferrara, D. Caput and G. Lefur, J. Mol. Graph., 1993, 11, 236.

9 V. Papadopoulos, H. Amri, H. Li, N. Boujrad, B. Vidic and M. Garnier, J. Biol. Chem., 1997, 272, 32129.

10 A. Verma, J. S. Nye and S. H. Snyder, Proc. Natl. Acad. Sci. U. S. A., 1987, 84, 2256.

11 J. Gatliff, D. East, J. Crosby, R. Abeti, R. Harvey, W. Craigen, P. Parker and M. Campanella, Autophagy, 2014, 10, 2279.

12 S. Galiegue, N. Tinel and P. Casellas, Curr. Med. Chem., 2003, 10, 1563.

13 E. Romeo, J. Auta, A. P. Kozikowski, D. Ma, V. Papadopoulos, G. Puia, E. Costa and A. Guidotti, J. Pharmacol. Exp. Ther., 1992, 262, 971.

14 G. Le Fur, M. L. Perrier, N. Vaucher, F. Imbault, A. Flamier, J. Benavides, A. Uzan, C. Renault, M. C. Dubroeucq and C. Gueremy, Life Sci., 1983, 32, 1839.

15 P. J. Marangos, J. Patel, J. P. Boulenger and R. Clark-Rosenberg, Mol. Pharmacol., 1982, 22, 26.

16 S. Z. Langer, S. Arbilla, S. Tan, K. G. Lloyd, P. George, J. Allen and A. E. Wick, Pharmacopsychiatry, 1990, 23, 103.

17 L. Jaremko, M. Jaremko, K. Giller, S. Becker and M. Zweckstetter, Science, 2014, 343, 1363.

18 N. Denora, V. Laquintana, M. G. Pisu, R. Dore, L. Murru, A. Latrofa, G. Trapani and E. Sanna, J. Med. Chem., 2008, 51, 6876.

19 G. Trapani, M. Franco, A. Latrofa, L. Ricciardi, A. Carotti, M. Serra, E. Sanna, G. Biggio and G. Liso. J. Med. Chem., 1999, 42, 3934.

20 N. Margiotta, N. Denora, R. Ostuni, V. Laquitana, A. Anderson, S. W. Johnson, G. Trapani and G. Natile, J. Med. Chem., 2010, 53, 5144. 
21 N. Margiotta, R. Ostuni, R. Ranaldo, N. Denora, V. Laquintana, G. Trapani, G. Liso and G. Natile, J. Med. Chem., 2007, 50, 1019.

22 C. Santini, M. Pellei, V. Gandin, M. Porchia, F. Tisato and C. Marzano, Chem. Rev., 2014, 114, 815.

23 P. K. Weiner, R. Langridge, J. M. Blaney, R. Schaefer and P. A. Kollman, Proc. Natl. Acad. Sci. U. S. A., 1982, $79,3754$.

24 S. Mukherjee, T. E. Balius and R. C. Rizzo, J. Chem. Inf. Model., 2010, 50, 1986.

25 P. T. Lang, S. R. Brozell, S. Mukherjee, E. F. Pettersen, E. C. Meng, V. Thomas, R. C. Rizzo, D. A. Case, T. L. James and I. D. Kuntz, RNA, 2009, 15, 1219

26 P. Csermely, R. Palotai and R. Nussinov, Trends Biochem. Sci., 2010, 35, 539.

27 B. Fresch and F. Remacle, Phys. Chem. Chem. Phys., 2014, 16, 14070.

28 B. Fresch and F. Remacle (2015). An Atomistic View of DNA Dynamics and Its Interaction with Small Binders: Insights from Molecular Dynamics and Principal Component Analysis. Single Molecular Machines and Motors: Proceedings of the 1st International Symposium on Single Molecular Machines and Motors, Toulouse 19-20 June 2013. C. Joachim and G. Rapenne. Cham, Springer International Publishing: 17.

\section{Acknowledgements}

The work of BF is supported by the Italian Ministero dell'Istruzione, Università e Ricerca - grant "Rita Levi Montalcini - 2013". The research of VG was supported by the University of Padova (Grants 60A04-0443, 60A043189 and 60A04-4015/15).

Electronic Supplementary Information (ESI) available: Experimental and computational details, crystallographic data, additional cytotoxicity data, values of the scoring functions, RESP partial atomic charges, cellular ROS levels, mitochondrial membrane potential and $\mathrm{O}_{2}$ consumption data, TEM images, body weight changes of treated mice, ligand poses and TSPO-complex structures. CCDC 1473112. See DOI: 10.1039/x0xx00000x 